\title{
An IoT application for environmental monitoring and control using Raspberry-Pi
}

\author{
Ravi Kiran Varma P ${ }^{\# 1}$, P L N Raju ${ }^{\# 2}$, M Priyanka ${ }^{\# 3}$ \\ \# MVGR College of Engineering (Autonomous) \\ Vizianagaram, Andhra Pradesh, India \\ ${ }^{1}$ ravikiranvarmap@gmail.com \\ ²plnraju@mvgrce.edu.in \\ 3priyanka.mandapati@gmail.com
}

\begin{abstract}
Raspberry Pi hardware board is an economical Internet of Things (IoT) hardware platform that can be used for wide variety of applications. This paper discusses the hardware, software platforms and implementation details of an IoT application for environmental monitoring and control using Raspberry PI 3 Model B. The DHT11 sensor is used to monitor temperature and humidity to further control the air conditioning system. The FC-37 sensor is used to detect the rainfall and further control the sprinkling system. The MQ135 sensor is used to detect the presence of hazardous gasses in the environment. Django web technology platform is used to design a dashboard for monitoring and controlling the things. MySQL database is used to log the sensor data and Fusion charts are used to display the trends in graphical form.
\end{abstract}

Keywords-IoT, Internet of Things, Embedded Systems, Raspberry PI, Sensors, Control.

\section{INTRODUCTION}

IoT is a technology that helps to establish internet connectivity between people and a wide variety of devices. IoT enables to monitor and control several parameters, take decisions, and automate the control system. The major advantages of IoT are continuous data logging, tracking the status of environment or the parameters under consideration, saving a lot of time and money. Raspberry Pi [1] is a popular 64-bit embedded hardware platform that is most suitable for IoT applications. This paper makes use of Raspberry Pi 3 model B hardware board along with few sensors in order to monitor and control environmental parameters and devices. The parameters that are monitored are temperature, humidity, rainfall, and hazardous gasses. The main goal of this work is to design an IoT for continuous monitoring and control of environmental parameters viz., temperature \& humidity, hazardous gases, and rain.

\section{RELATED WORK}

Anwaar and Shah[2] compared raspberry pi with other technological inventions like Smart Phone, tablet computer, smart TV, and Laptop. Their outcomes demonstrate that raspberry pi consumes less power and computation jobs can be accomplished in a better vitally effective manner. In this work he used hardware devices like tablet Pc, laptop, smart TV and raspberry Pi. Here energy efficiency is calculated in kilojoules per hour. The authors have compared the power consumptions of all the computing devices. They observed that the desktop consumes 82 watts, a laptop, a smart phone consumes 50 and 5 watts respectively. Whereas a raspberry pi system only consumed 2.25 watts. Therefore raspberry pi is more energy efficient than the other computing devices.

Zhao et al.[3], explored the application of raspberry pi system as a server along with other laptops in the same network. They have established client server communication using Wi-Fi, ZigBee protocols within localhost for remote file sharing. Samba is an open source software used in this project for file and print services. Raspbian Operating Systems was used for raspberry pi project development. Varma et al. used Rabbit processor as an embedded web server and also implemented a Denial of Service attack detection mechanism [4]. Sandeep et al.[5]have proposed this automation system to control machines in laboratories with high mobility and security.Electronic devices were interfaced to the raspberry pi and were remotely controlled through weaved fabric cloud platform. The General Purpose Input Output (GPIO) pins of the raspberry pi board were controlled using Webiopi software. The raspberry pi was interfaced to Digiduino board for amplification, in order to connect the relay switches to control the external devices.

Ibrahim et al.[6] developed a low-cost environmental monitoring system by making use of the raspberry pi computing board. Sensors used in this work are temperature sensor TMP36, Humidity DHT22, CO concentration using MQ-7, Earthquake thin-film-piezoelectric sensor LDT0028K. Pavithra and Balakrishnan[7] proposed an efficient system for monitoring and controlling the home appliances via World Wide Web. In this work communication takes place through internet gateway by using protocols like Zigbee, WiFi etc. In addition to that fire alarm system has been established. Home appliances used are lights, and fan. Sensors used are IR sensor for lights, PIR sensor for fans and Fire detection sensor for fire. These sensors are connected and 
interfaced through raspberry pi and can be controlled through our mobile phones. WiFi provides the medium for configuration which can be configured to make security services. Different web pages are created for different rooms to access appliances separately. One of the advantages is that this system can be monitored and controlled from any OS in mobile phones.

Daniel et al.[8]proposed a system to control the temperature in anautomated and efficient way using Fuzzy logic. Here prototype consumes data from sensors and IoT platform. The main idea is to get temperature on outdoor and basing on that system is designed to control indoor temperature using IoT. Hardware components used in this work are a TMP36 temperature sensor, MCP3008 analogic-to-digital converter, 2 yellow LEDs, 2 red LEDs, and 1 RGB LED,Several resistors.Chowdhury et al.[9]designed a raspberry pi based visitor notification system. They have interfaced a camera to take a picture of the visitor and notify through twitter. WiFi communication was used to interface the camera and Infra-Red (IR) sensor for human presence sensing. Raspberry pi was configured as an Internet server to remotely communicate the information to anywhere the user wants. Street lights were automated through IoT technology using raspberry pi by Leccese et al. in [10]. Aksh et al. [11] designed a raspberry pi project to control wheel chair through IoT using WebIOpi framework. Ray [12] has done a similar work to monitor MISSENARD index using Arduino hardware platform and ThingSpeak and Plotly cloud software platform. Other interesting applications of Raspberry pi include controlling a car remotely [13], home automation [14]. Security is one the major concern in IoT projects. Intrusion Detection Systems (IDS) and Firewalls [15][16][17][18][19][20]takes the front seat in protecting the network perimeter along with host based security tools.

\section{III.EnVironmental Monitoring ANd Control Using RASPberry PI}

This work proposes a smart environmental parameters monitoring and control system using Raspberry Pi 3 Model B. Three different sensors are used to monitor the temperature and humidity, rainfall, and hazardous gasses. The block diagram is as shown in Fig.1. A picture of the project hardware is shown in Fig.2.

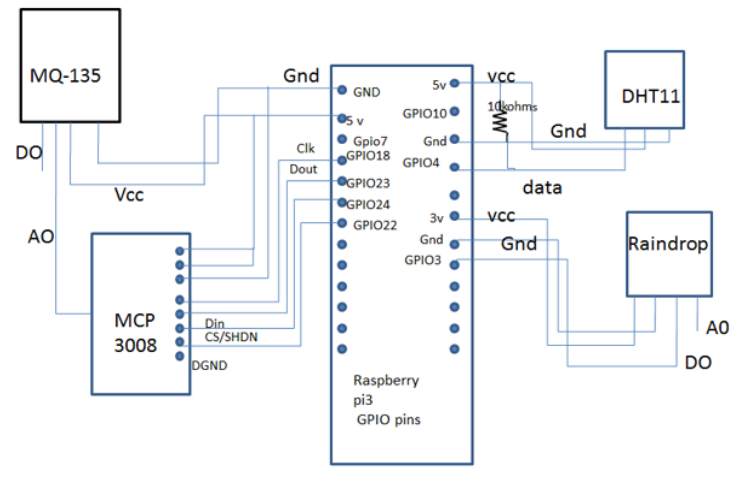

Fig.1 The proposed system block diagram
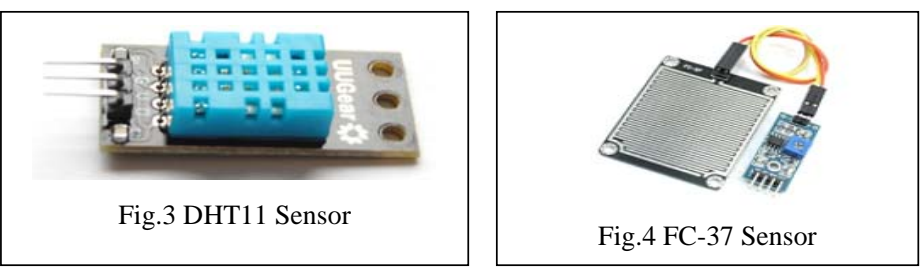

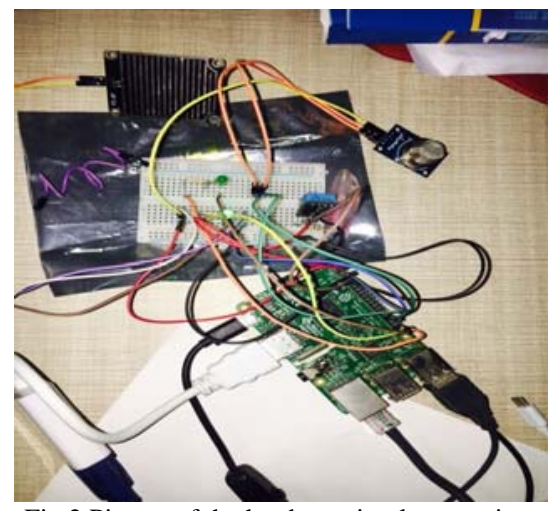

Fig.2 Picture of the hardware implementation

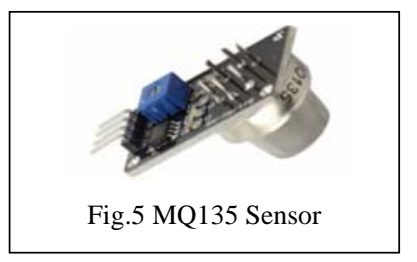

\section{A. About the sensors used in the system}

DHT-11 is a temperature and humidity sensor, shown in Fig.3. The advantage of this sensor is it is highly reliable, low price, good quality, faster response time, and inference free design. This sensor can measure temperatures ranging from 0 to $50^{\circ} \mathrm{C}$ with an error of plus or minus $2^{\circ} \mathrm{C}$. The range of humidity measure that DHT11 can support is 20 to $90 \%$ RH with an allowable error of $5 \%$. It can directly provide a digital output with its own serial communication protocol. It can operate at a low supply voltage of minimum $3 \mathrm{~V}$ up to $5.5 \mathrm{~V}$. It has only three pins, for Vcc, data, and ground. It uses Negative Temperature Coefficient (NTC) based temperature sensing component, and moisture substrate based humidity sensing component.

FC-37 is a raindrop sensor, shown in Fig.4. It contains two parts, one board is the electronic board and the other one is the rain drop collecting board. It has both analog as well as digital output. The digital output is one bit binary to indicate if there is rain or not. It operates on a $5 \mathrm{~V}$ supply. The digital output remains at a high level when the surface is dry and output goes to a low level when the surface is wet. 
MQ135 is a gas sensor (Fig.5) that can detect harmful gasses including smoke, Sulphide, Ammonia, Benzene, Carbon dioxide, and alcohol. MQ135 indicates the type of gas in the form of an analog signal and an Analog-toDigital converter MCP3008 is used to convert it to digital and interface to the raspberry pi.

\section{B. Software platform}

Raspbian Operating system is used along with apache web server. Django Python web framework is used for the web interface development. The advantage of Django is that it is a fast, secure and scalable platform. Only free and open source software are used in the design of the proposed system. MySQL is used for the database interface to the system. Fusion charts are used in visualizing the sensor data in the form of graphs. Fig. 6 shows the installation steps in the raspberry pi.

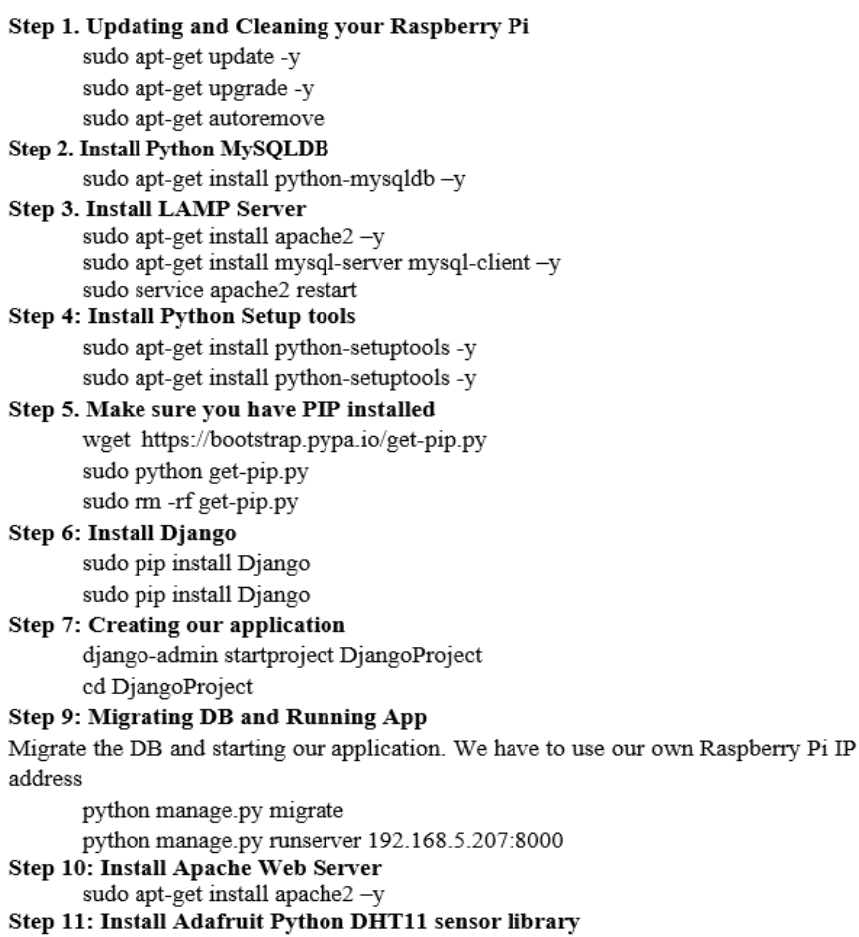

Fig.6 Installation steps of software

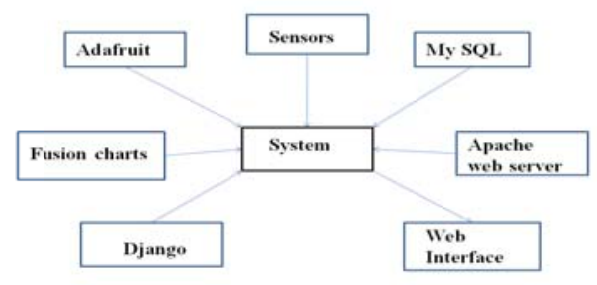

Fig.7 Software Block Diagram

C. Algorithm

The algorithm that is developed for an IoT application for environmental monitoring and control is as shown in Algorithm.1.

Algorithm 1

Step1: start

Step2: declare variables humidity, temperature, gas,gas1, state, rain, sprinkler, AC.

Step 3: loop

Step 4: reads humidity and temperature values from dht11

Step 5: if temperature $>40$ ac $=$ on

else ac $=$ off 
Step 6: reads state from fc-37

Step 7: if state $==1$ rain= rainfall detected and sprinkler $=$ off

else rain $=$ not detected sprinkler $=$ on

Step 8: reads gas value from $\mathrm{mq}-135$

Step 9: if gas $<180$ :

$$
\text { gas1="normal gas " }
$$

else if gas $<400$ and gas $>380$ :

$$
\text { gas1= "co2 " }
$$

else if gas $<440$ and gas $>400$ :

gas1= "benzene "

else if gas $<700$ and gas $>680$ :

gas1= "alcohol "

Step 10: return temperature, humidity, rain, ac, sprinkler, gas, gas1 to webpage

Step 11: end loop

Step 12: stop

\section{IV.RESULTS}

In the Login page authentication is done. If the user is validated, the main page will be displayed, otherwise it will redirect to login page saying invalid user. The screen shot of login page is shown in Fig.8 All sensor results are displayed on this page. Temperature, humidity and gas concentration values are shown in Fig.9. The web page is dynamic and the refresh cycle is set to few seconds that can be programmed as required.

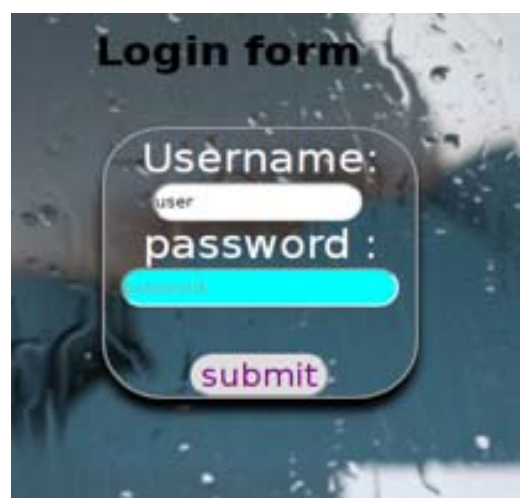

Fig. 8 Login Page for authentication

The code snippet for logging the sensor data using the MySQL data base is shown below.

fromdjango.db import models

fromdatetime import datetime

class Weather(models.Model):

temperature $=$ models.DecimalField $($ max_digits $=5$,decimal_places $=2)$

humidity $=$ models.DecimalField $($ max_digits $=5$,decimal_places $=2$ )

rainfall $=$ models.CharField(max_length $=20$ )

gas $=$ models.CharField $\left(\max \_\right.$length $\left.=20\right)$

date $=$ models.DateTimeField(default $=$ datetime.now,blank=True)

A sample data base is shown in Fig.10. A sample graph generated by fusion chart is shown in Fig.11. 


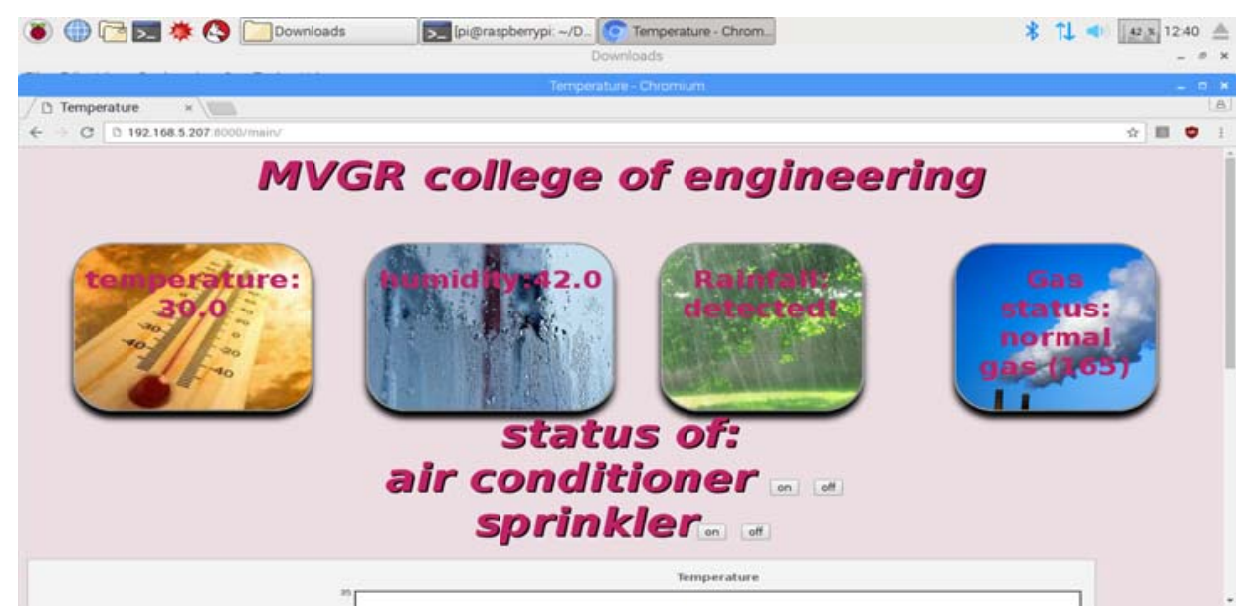

Fig. 9 Web Interface

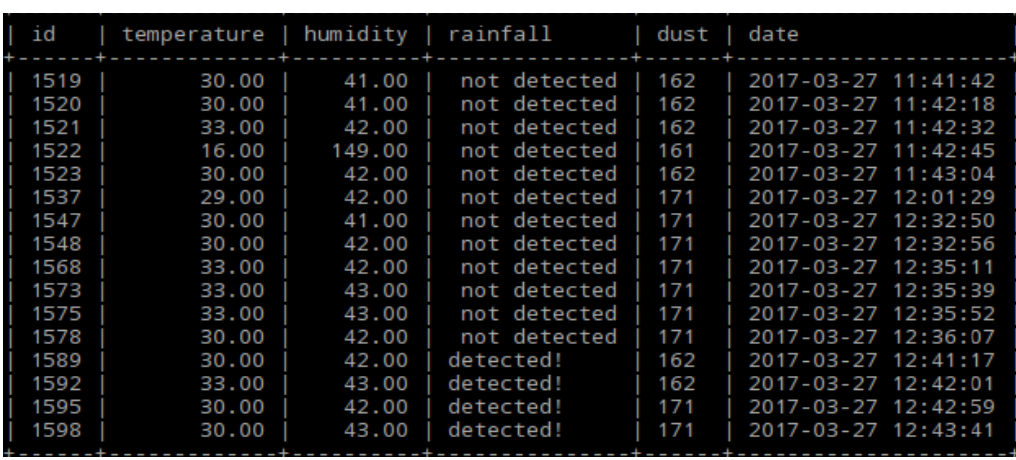

Fig.10 Sensor Database

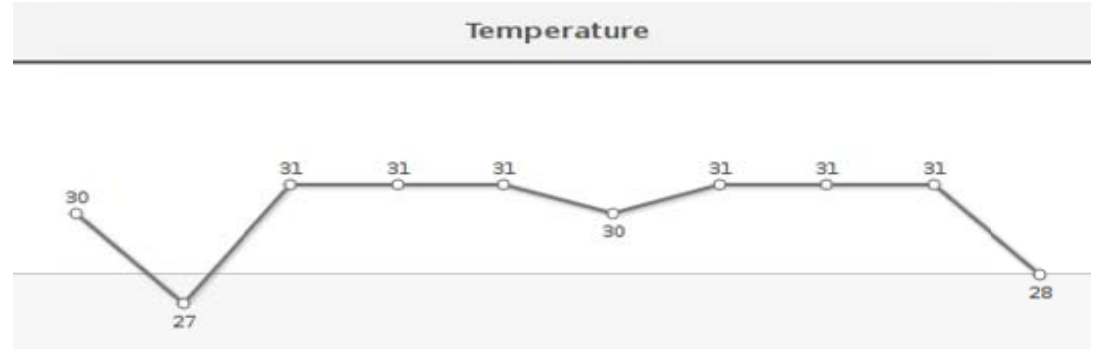

Fig. 11 Output of the Fusion Chart for temperature data

\section{CONCLUSION}

The proposed IoT application for environmental monitoring and control using Raspberry PI provides an economical user-friendly web interface for monitoring and controlling IoT sensors. It is an automated, scalable, and cheaper solution. We can see the results from anywhere in the world through the web interface. Manual as well as automated control of output devices viz., sprinklers and the air conditioner is implemented. In this work all results are stored in a database which can be viewed through charts (Fusion Charts). For that we used a framework called Fusion Charts which can be downloaded from the official Website. Fusion Charts enables us to get the data from the database with few lines of code and directly integrates with the required chart. Chart look and feel can be changed by simply selecting the theme. Further work is needed to implement this on the android mobile platform. 


\section{References}

[1] R. P. foundation, “Teach, Learn and Make with Raspberry Pi,” [Online]. Available: https://www.raspberrypi.org/. [Accessed 15 Jan 2017].

[2] Waqas Anwaar and Munam Ali Shah, "Energy Efficient Computing: A Comparison of Raspberry PI with Modern Devices," International Journal of Computer and Information Technology, vol. 04, no. 02, pp. 410-413, March 2015.

[3] Cheah Wai Zhao, Jayanand Jegatheesan and Son Chee Loon, “Exploring IOT Application Using Raspberry Pi,” International Journal of Computer Networks and Applications, vol. 2, no. 1, pp. 27-34, February 2015.

[4] Ravi Kiran Varma P and Valli Kumari V, “A Security Framework For Ethernet Based Embedded Web Server,” International Journal of Embedded Systems and Applications, vol. 2, no. 2, pp. 17-27, 2012.

[5] V Sandeep, K Lalith Gopal, S Naveen, A Amudhan and L S Kumar, "Globally Accessible Machine Automation Using Raspberry Pi," in International Conference on Advances in Computing, Communications and Informatics (ICACCI), Kochi, India, 2015.

[6] Mohannad Ibrahim, Abdelghafor Elgamri, Sharief Babiker and Ahmed Mohamed, "Internet of Things based Smart Environmental Monitoring using the Raspberry-Pi Computer," in Fifth International Conference on Digital Information Processing and Communications (ICDIPC), Sierre, Switzerland, 2015.

[7] Pavithra D and Ranjith Balakrishnan, "IoT based Monitoring and Control System for Home Automation,” in Proceedings of 2015 Global Conference on Communication Technologies, Thuckalay, India, 2015.

[8] Daniel Meana-Llorián, Cristian González García, B Cristina Pelayo G-Bustelo, Juan Manuel Cueva Lovelle and Nestor GarciaFernandez, "The fuzzy logic and the Internet of Things to control indoor temperature regarding the outdoor ambient conditions," Future Generation Computer Systems, vol. Article In Press, pp. 1-10, 2016.

[9] Md Nasimuzzaman Chowdhury, Md Shiblee Nooman and Srijon Sarker, "Access Control of Door and Home Security by Raspberry Pi Through Internet,” International Journal of Scientific \& Engineering Research, vol. 4, no. 11, pp. 550-558, November 2013.

[10] Fabio Leccese, Marco Cagnetti and Daniele Trinca, "A Smart City Application: A Fully Controlled Street Lighting Isle Based on Raspberry-Pi Card, a ZigBee Sensor Network and WiMAX,” Sensors, vol. 14, pp. 24408-24424, 2014.

[11] Akash S A, Akshay Menon, Arpit Gupta, P. M. Md Waheeb Wakeel and P Meena, "A novel strategy for controlling the movement of a Smart Wheelchair using Internet of Things," in 2014 IEEE Global Humanitarian Technology Conference- South Asia Satellite (GHTC-SAS), Trivandrum, 2014

[12] P. P. Ray, "Internet of Things cloud enabled MISSENARD index measurement for indoor occupants," Measurement, vol. 92, no. 2016, pp. 157-165, 2016.

[13] Gurjashan Singh Pannu, Mohammad Dawud and Pritha Gupta, "Design and Implementation of Autonomous Car using Raspberry Pi," International Journal of Computer Applications, vol. 113, no. 9, p. 0975 - 8887, 2015.

[14] Vladimir Vujovic and Mirjana Maksimovic, "Raspberry Pi as a Sensor Web node for home automation," Computers and Electrical Engineering, vol. 44, no. 2015, pp. 153-171, 2015.

[15] Ravi Kiran Varma P and Valli Kumari V, "Feature Optimization and Performance Improvement of a Multiclass Intrusion Detection System Using PCA and ANN,” International Journal of Computer Applications, vol. 44, no. 13, pp. 4-9, 2012.

[16] Ravi Kiran Varma P, Valli Kumari V and Srinivas Kumar S, “Ant Colony Optimization-based Firewall Anomaly Mitigation Engine,” Springerplus, vol. 5, no. 1, pp. 1-32, 2016.

[17] Ravi Kiran Varma P, Valli Kumari V and Srinivas Kumar S, "Feature selection using relative fuzzy entropy and ant colony optimization applied to real-time intrusion detection system,” Procedia Computer Science, vol. 85, no. 2016, pp. 503-510, 2016.

[18] P Ravi Kiran Varma, V Valli Kumari and S Srinivas Kumar, "Packet Filter Firewall Rule Anomalies and Mitigation Techniques: A Technical Review,” CiiT International Journal of Networking and Communication Engineering, vol. 9, no. 4, pp. 101-108, 2017.

[19] P. R. K. Varma, V. V. Kumari and S. S. Kumar, "Application of Rough Sets and Ant Colony Optimization in feature selection for Network Intrusion Detection,” International Journal of Applied Engineering Research, vol. 10, no. 22, pp. 43156-43163, 2015.

[20] Ravi Kiran Varma P, Valli Kumari V and Srinivas Kumar S, "A Novel Rough Set Attribute Reduction based on Rough Sets and Ant Colony Optimization,” International Journal Intelligent Systems Technologies and Applications, vol. 14, no. 3/4, pp. 330-353, 2015.

\section{AUTHOR'S PROFILE}

Ravi Kiran Varma Penmatsa is currently working as Associate Professor at Maharaj

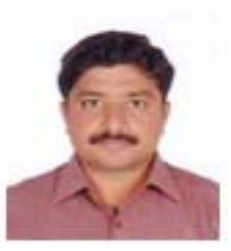
Vijayaram Gajapathi Raj College of Engineering, Vizianagaram, AP, India. He has over 15 years of experience. He has submitted his Ph.D thesis to JNT University, Kakinada in the area of network security and soft computing. He is the founder coordinator of M.Tech (Computer Networks and Information Security) course at MVGR and is involved actively in the curriculum development and laboratory establishments of the course. He has achieved Cisco Certified Network Associate (CCNA), EC-Council Certified Ethical Hacker (CEH) certifications. He is an authorized instructor for Cisco and EC-Council academies. He has achieved Wipro Mission 10X advanced certification in High Impact Teaching Skills. He is a member of professional bodies like IEEE, ACM, CSI, IE, and ISTE. He is serving as faculty sponsor for ACM student chapter of MVGR. He has published several research articles in international journals and conferences of repute. His areas of interest include Network Security, Soft Computing, Machine Learning, Artificial Intelligence, Embedded Systems, and IoT. 


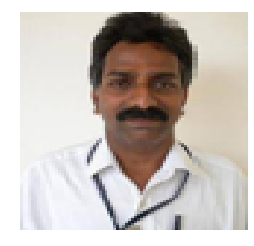

P L N Raju is currently working as Assistant Professor of CSE department, MVGR College of Engineering, Vizianagaram since 2003. He has over 16 years of teaching and industrial experience. He has completed M.Tech (IT) from Andhra University. He is a member of professional societies like CSI, ISTE, and IE. Apart from academics he also served as committee member of E-Service, Magazine, and Anti-Ragging. He has attended several workshops and FDP's including NPTEL courses. His areas of interest include Information Security and Networking.

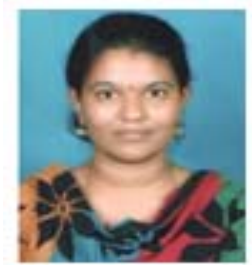

Ms. M Priyanka is presently working as Assistant Professor of CSE at MVGR College of Engineering, Vizianagaram, AP since 2009. She has completed her M.Tech (CSE) from Andhra University. She is a member of the Institute of Engineers and IAENG. Her areas of interest includes Software Engineering, Computer Networks and Internet of Things. She has attended several continuing education programs including workshops and FDP's. 